\title{
Mucinous Cystadenomas in Liver: Management and Origin
}

\author{
D. Erdogan ${ }^{a} \quad$ J. Kloek ${ }^{a} \quad$ W.H. Lamers ${ }^{b} \quad$ G.J.A. Offerhaus ${ }^{c} \quad$ O.R.C. Busch ${ }^{a}$ \\ D.J. Gouma ${ }^{a}$ T.M. van Gulik ${ }^{a}$ \\ Departments of ${ }^{\text {a Surgery, }}{ }^{\mathrm{b}}$ Anatomy and Embryology, and ${ }^{\mathrm{C}}$ Pathology, Academic Medical Center, \\ University of Amsterdam, Amsterdam, The Netherlands
}

\section{Key Words}

Cystadenoma - Cystadenocarcinoma - Gonadal epithelium • Ovarian stroma $\cdot$ Embryo $\cdot$ Liver

\begin{abstract}
Background: Mucinous cystadenomas of the liver are rare cystic neoplasms. The aim of this study was to assess management of a consecutive series of patients who underwent laparotomy for a suspected cystadenoma or cystadenocarcinoma. Secondly, the origin of ovarian stroma (OS) in mucinous liver cystadenomas was examined during early embryonic development. Patients and Methods: Patients diagnosed with mucinous liver cystadenomas or cystadenocarcinoma between 1994 and 2009 were included. Pathology specimens of patients who had undergone resection were reviewed for OS. Furthermore, in human embryos, morphology of the peritoneal epithelium and the position of the gonads in relation to the embryonic liver, pancreas and spleen were examined. Results: 15 surgically treated patients (13 female, 2 male) with hepatic tumors were eventually diagnosed with mucinous liver cystadenomas (12) or cystadenocarcinomas (3). OS was present in all female patients with mucinous cystadenoma or cystadenocarcinoma. The 2 male patients were rediagnosed as intraductal papillary mucinous neoplasm (IPMN) or cystadenocarcinoma with features of IPMN.
\end{abstract}

In human embryos, preceding their 'descent', the gonads are situated directly under the diaphragm, dorsal to the liver, the tail of the pancreas and the spleen, but separated from these organs by the peritoneal cavity. In contrast to the peritoneal epithelium elsewhere, the cells covering the gonads show an activated morphology. Conclusion: For the diagnosis of mucinous liver cystadenoma, the presence of $\mathrm{OS}$ is prerequisite. This may be explained by the common origin of cystadenoma and OS in epithelial cells that cover the embryonic gonads in early fetal life.

Copyright $\odot 2010$ S. Karger AG, Basel

\section{Introduction}

Mucinous cystadenomas of the liver are rare cystic neoplasms that usually arise in the liver or extrahepatic bile ducts and may show malignant degeneration into cystadenocarcinomas. These lesions tend to occur more often in middle-aged women and account for less than $5 \%$ of all the cysts found in the liver. On histopathological examination, two groups can be distinguished according to the presence or absence of mesenchymal stroma that resembles ovarian stroma (OS) [1-3].

The presence of OS in mucinous liver cystadenomas suggests a correlation with ovarian mucinous cystic neo-

\section{KARGER}

Fax +4161306 1234

E-Mail karger@karger.ch

www.karger.com
(C) 2010 S. Karger AG, Basel

0253-4886/10/0271-0019\$26.00/0

Accessible online at:

www.karger.com/dsu
T.M. van Gulik, MD

Department of Surgery, Academic Medical Center

Meibergdreef 9, NL-1105 AZ Amsterdam (The Netherlands)

Tel. +31 20566 5570, Fax +31 206976621

E-Mail t.m.vangulik@amc.uva.nl 
plasms. It has been suggested that the near location of the liver to the gonads during embryonic development is responsible for migration of gonadal cells into the liver and the finding of OS in these lesions [4].

In this study, management is assessed of a consecutive series of patients who underwent laparotomy for a suspected cystadenoma or cystadenocarcinoma. Secondly, the regional differences in morphology of the peritoneal cavity is examined in human embryos, especially in relation with the cells covering the gonads, in order to investigate the presence of OS in liver mucinous cystadenomas.

\section{Patients and Methods}

Patients with mucinous liver cystadenomas and cystadenocarcinomas who presented at the Academic Medical Center in Amsterdam, The Netherlands, between April 1994 and August 2009, were selected from the database of the Department of Surgery. The surgical resection specimens of these patients were reassessed by a single pathologist specialized in hepatobiliary pathology (G.J.A.O.) to review the presence of OS.

Human embryos, part of the collection of the Department of Anatomy \& Embryology at the Academic Medical Center, were used, fixed in $4 \%$ buffered formaldehyde and stained with hematoxylin and azophloxin. Regional differences in morphology of the epithelium of the peritoneal cavity and the position of the gonads in relation to the embryonic liver, pancreas and spleen were examined at 5-8 weeks of development.

\section{Results}

\section{Patient Features}

Clinical Presentation

15 surgically treated patients (13 female, 2 male) with hepatic tumors were eventually diagnosed with mucinous liver cystadenomas [12] or cystadenocarcinomas [3]. Symptoms included abdominal pain/discomfort in $8 \mathrm{pa}-$ tients. Five patients presented with biliary obstruction. In 2 patients, the hepatic lesions were incidental findings. The mean age at presentation was 45 years (range 27-70).

\section{Imaging Studies}

The most frequently utilized diagnostic imaging tests were ultrasonography and contrast-enhanced computed tomography. On ultrasonography, cystadenomas appeared as anechoic, multiseptated structures. On computed tomography, these lesions were usually multicystic and multiseptated (fig. 1). The mean size and standard deviation of the liver lesions was $8.4 \pm 5.3 \mathrm{~cm}$ (range $3-19)$.

\section{Surgical Therapy}

All 15 patients underwent resection. The surgical procedures in the liver included left hemihepatectomy in 3 patients. One patient underwent extended right hemihepatectomy, 9 patients underwent enucleation of the lesion and 2 patients underwent left hemihepatectomy combined with hilar resection including the hepatic duct confluence. Postoperative complications occurred in 2 patients (subphrenic abscess and transient kidney failure). There was no postoperative mortality.

\section{Histopathological Assessment}

In 13 female patients, histopathological examination revealed a mucinous cystadenoma in 12 patients and a cystadenocarcinoma in 1 patient. In the 12 patients with cystadenoma, microscopical examination revealed cysts with a lining of non-ciliated, cuboidal epithelial cells. The subepithelial layer consisted of densely organized, spindle-shaped cells resembling OS. In the female patient with cystadenocarcinoma, the neoplasm consisted of epithelium with dysplastic features and cellular invasion in the presence of OS.

The 2 male patients underwent resection under the presumptive diagnosis of mucinous cystadenocarcinoma. In 1 of these patients, the diagnosis of mucinous cystadenocarcinoma was confirmed, probably in the presence of biliary intraductal papillary mucinous neoplasm (IPMN). In the other male patient, the histopathological diagnosis was IPMN (without signs of malignancy).

\section{Embryological Investigations}

In the 5th week of pregnancy, the gonads have developed from the celomic epithelium of the urogenital fold, the primordial germ cells and the local mesenchyme. At this stage, the gonads are localized just under the developing diaphragm, the right gonad lying dorsolaterally to the liver and the left gonad dorsolaterally to the pancreas and spleen [5]. In the embryo, the stomach and the small bowel are topographically further away from the gonads, as the dorsal mesentery separates the stomach from the left gonad, while the intestines are 'ventral' organs in the embryo. These topographical relations start to change after the 8th week when the gonads descend to their definitive location in the pelvis (fig. 2).

The peritoneal cells covering the gonads differ from those covering the liver, pancreas and spleen in that their shape is remarkably rounded up compared to the typical flat epithelium elsewhere in the celomic cavity (fig. 3). This morphology suggests activation of the peritoneal epithelial cells covering the gonads. 


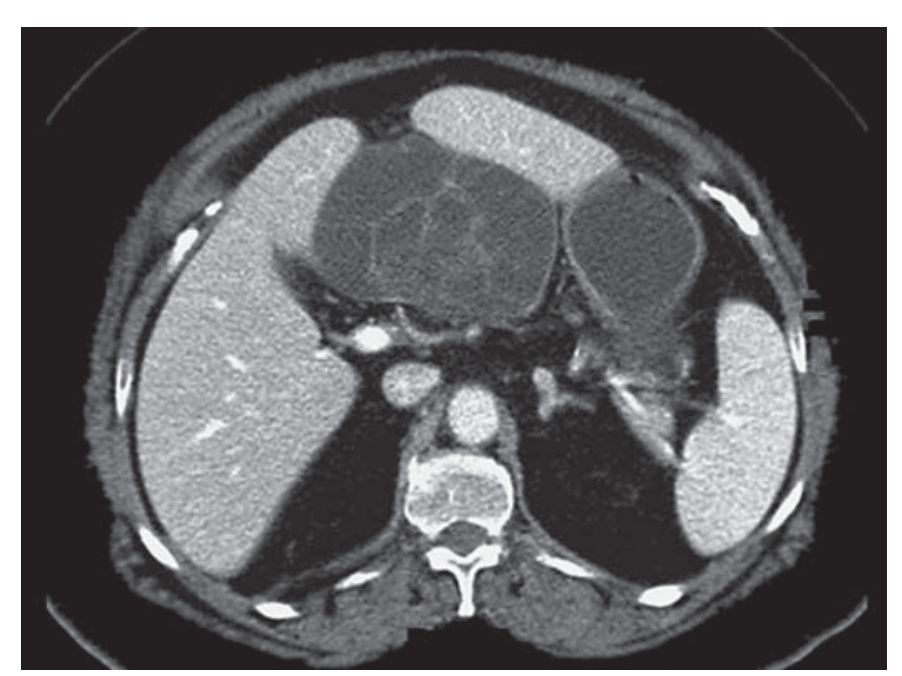

Fig. 1. Abdominal CT demonstrating a cystic lesion with internal septations located in the liver. Histopathological examination of the resection specimen revealed a mucinous cystadenomas with ovarian stroma.

\section{Discussion}

Patients with mucinous cystadenomas of the liver showing OS are usually females in their reproductive period and are younger compared to patients with cystadenomas without OS. Cystadenomas are rarely seen in male patients and then lack OS $[1,3]$. Patients usually present with abdominal pain, discomfort and jaundice when biliary obstruction is present. Cystadenomas may give rise to confusion on imaging studies as these are easily mistaken for simple cysts, hydatid cysts or Caroli disease. Typical features on imaging studies include internal septations and an irregular, thickened cyst wall with polypoid protrusions. Jaundice in combination with a cystic lesion is usually a harbinger of infiltrating cystadenocarcinoma $[6,7]$. Although in the past, cystadenomas of the liver have been treated by internal drainage or partial resection, the therapy of choice is now considered complete surgical excision because of potential malignant degeneration into cystadenocarcinomas. However, it is still uncertain whether cystadenocarcinoma is a newly developed cancer or is derived from a cystadenoma [8].

The lining of mucinous liver cystadenomas is microscopically composed of biliary-type mucus-secreting cuboidal or columnar epithelium. The dense subepithelial stroma with spindle cells has the same features as normal OS $[1,2]$. The fact that this tissue expresses receptors for female sex hormones in part of the patients explains their

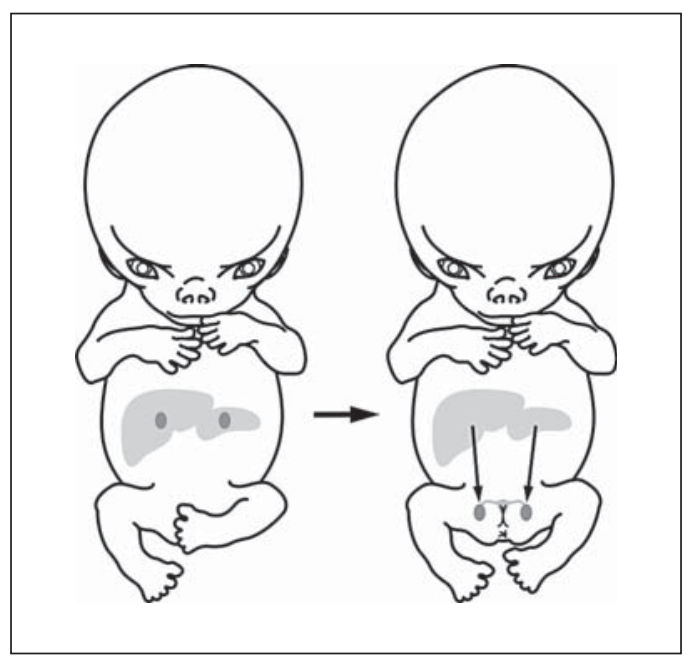

Fig. 2. Representation of gonads descending to their definitive location in the pelvis after 8 weeks of gestation. Initially, the right gonad lies dorsolaterally to the liver and the left gonad dorsal to pancreatic tail and spleen.

remarkable growth during pregnancy [9]. The mean age of patients with malignant degeneration of a mucinous cystadenoma was reported to be approximately 17 years higher in comparison to patients without malignant degeneration [3].

Imaging modalities are still not sufficient to reliably distinguish mucinous liver cystadenoma and cystadenocarcinoma. Furthermore, it is preoperatively difficult to differentiate these cystic tumors from IPMN, which are histopathologically characterized by the lack of OS, excessive amounts of mucin production, and a predominantly intraductal growth pattern [10]. In our series, 1 patient diagnosed with cystadenocarcinoma was eventually found to harbor IPMN in the tumor, whereas in another patient suspected of mucinous cystadenocarcinoma, features of IPMN were found, however without signs of malignant degeneration. As previously reported, mucinous liver cystadenoma and cystadenocarcinoma without OS should be regarded as IPMN [11].

The similarity in clinicopathological features of mucinous cystadenomas with OS originating in liver, pancreas, or retroperitoneum [12] with mucinous neoplasms of the ovary, suggests a correlation. A similar correlation has been suggested of mucinous neoplasms of the peritoneal cavity (pseudomyxoma peritonei) and mucinous neoplasms of the ovary [13]. The hypothesis that ectopic OS originates from the gonads is not only based on the observation that the most frequently affected organs (i.e. 
Fig. 3. a Overview of a 5-week-old human embryo showing the left upper abdomen. $\mathbf{b}$ and $\mathbf{c}$ show the insets indicated in $\mathbf{a}$ and depict high-power views of the peritoneal epithelium covering the gonad and the spleen (b) or stomach (c). Note that the cells of the gonadal epithelium protrude from the surface, whereas the peritoneal cells covering the stomach form a continuous flat layer. The bright red cells with a nucleus are embryonic erythrocytes. Their presence in the peritoneal cavity is artifactual. $\mathrm{A}=$ Aorta; $\mathrm{Go}=\operatorname{gonad} ; \mathrm{L}=$ liver; $\mathrm{M}=$ mesonephros; $\mathrm{P}=$ pancreas; $\mathrm{S}=$ stomach; $\mathrm{Sp}=$ spleen .
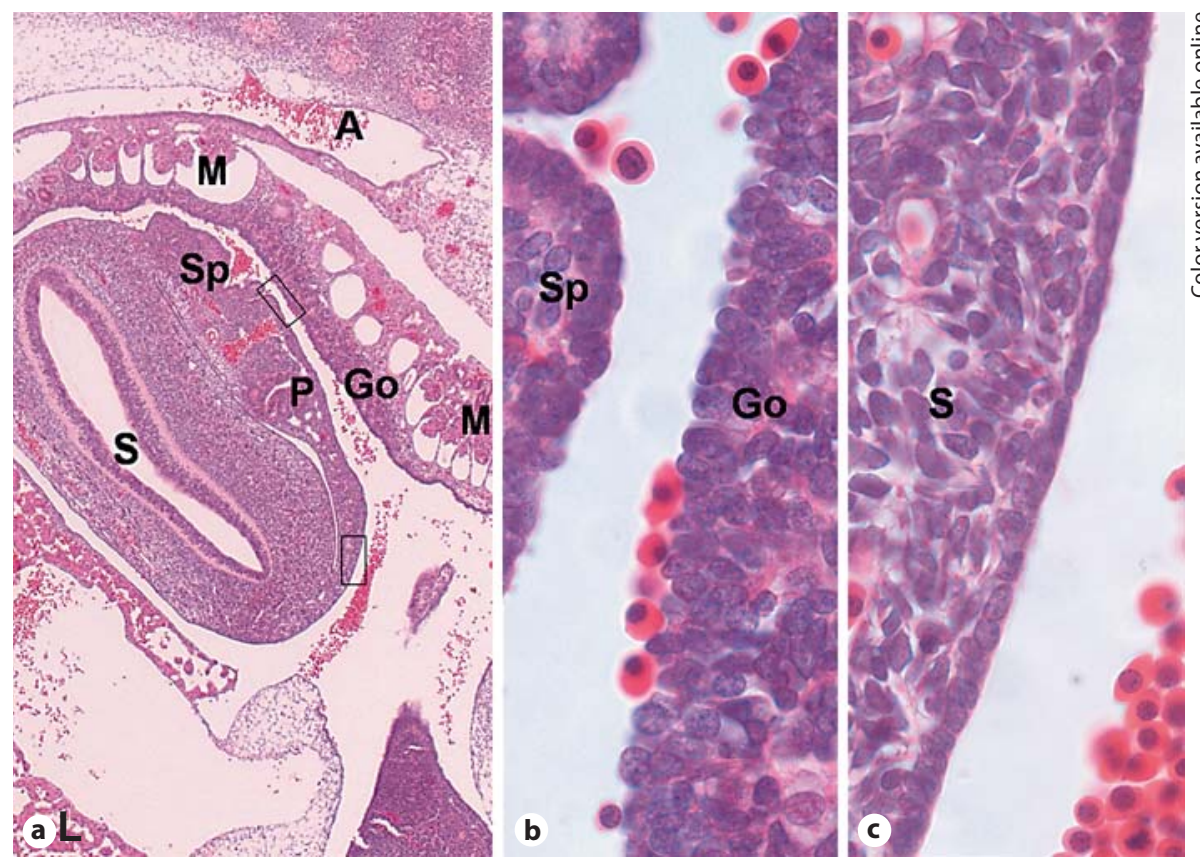

spleen, liver and pancreas) are in close topographical contact with the embryonic gonads, but also on the finding that the peritoneal surface epithelium of the embryonic gonads are distinct in that the gonads are lined with bulging cells as opposed to the typical flattened celomic epithelium. This special morphology suggests that the epithelial cells covering the gonads are activated and have the ability to detach from the gonadal surface, then 'cross' the peritoneal cleft and attach to the peritoneal surface of nearby organs [14].

Fusion of the testes or the ovaria with the liver, adrenals or especially with the spleen has often been documented by pediatric surgeons [15-17]. Such fusions probably arise between the 5th and 8th week of development. In this period, the gonads are located just below the diaphragm, at the level of the dorsocranial side of the liver, the tail of the pancreas, and the spleen [5]. It has recently been shown that epithelial cells express hepatocyte-growth factor/scatter factor (HGF/SF) [18]. As the name 'scatter factor' suggests, exposure to this growth factor leads to detachment of epithelial cell connection and migration of cells. It is important to note that the expression of HGF/SF in the epithelial cells of the ovary ceases when follicles become identifiable. In case of the testes, the expression of HGF/SF is still ongoing. This difference in expression between the developing testes and the developing ovary may explain why fusion of a gonad with liver, spleen or adrenals appears to be confined to the testes.

In conclusion, the treatment of choice for mucinous cystadenoma of the liver is radical excision of the lesion, taking into account the risk of malignant transformation into cystadenocarcinoma. Examination of embryos suggests that during the embryonic period, the epithelial cells of the gonads detach from their tissue boundaries and migrate into the surface of nearby organs such as the liver. Mucinous liver cystadenomas and cystadenocarcinomas without OS are probably a different entity.

References

Erdogan/Kloek/Lamers/Offerhaus/ Busch/Gouma/van Gulik 
5 Moore KL, Persaud TVN: The Developing Human, ed 5. Philadelphia, Saunders, 1993.

-6 Erdogan D, Busch OR, Rauws EA, et al: Obstructive jaundice due to hepatobiliary cystadenoma or cystadenocarcinoma. World J Gastroenterol 2006;12:5735-5738.

7 Thomas KT, Welch D, Trueblood A, et al: Effective treatment of biliary cystadenoma. Ann Surg 2005;241:769-773.

-8 Kubota E, Katsumi K, Iida M, et al: Biliary cystadenocarcinoma followed up as benign cystadenoma for 10 years. J Gastroenterol 2003;38:278-282.

-9 Vogt DP, Henderson JM, Chmielewski E: Cystadenoma and cystadenocarcinoma of the liver: a single-center experience. J Am Coll Surg 2005;200:727-733.
10 Kloek JJ, van den Gaag N, Erdogan D, et al: A comparative study of intraductal papillary neoplasia of the biliary tract and pancreas (submitted).

11 Zen Y, Fujii T, Itatsu K, et al: Biliary cystic tumors with bile duct communication: a cystic variant of intraductal papillary neoplasm of the bile duct. Mod Pathol 2006;19:12431254.

12 Subramony C, Habibpour S, Hashimoto LA: Retroperitoneal mucinous cystadenoma. Arch Pathol Lab Med 2001;125:691-694.

13 Galani E, Marx GM, Steer CB, et al: Pseudomyxoma peritonei: the 'controversial' disease. Int J Gynecol Cancer 2003;13:413-418.
4 Erdogan D, Lamers WH, Offerhaus GJ, et al: Cystadenomas with ovarian stroma in liver and pancreas: an evolving concept. Dig Surg 2006;23:186-191.

15 Nimkin K, Kleinman PK, Chappell JS: Abdominal ultrasonography of splenogonadal fusion. J Ultrasound Med 2000;19:345-347.

16 Basar M, Erdogan S, Aydoganli L, et al: Aberrant adrenal cortical tissue adjacent to immature testis. Arch Ital Urol Androl 1997;69: 141-142.

17 Duncan WL Jr, Barraza MA: Splenogonadal fusion: a case report and review of literature. J Pediatr Surg 2005;40:e5-e7.

18 Ricci G, Catizone A, Galdieri M: Pleiotropic activity of hepatocyte growth factor during embryonic mouse testis development. Mech Dev 2002;11:819-828. 